\title{
A TERMINATION PROTOCOL FOR SIMPLE NETWORK PARTITIONING IN DISTRIBUTED DATABASE SYSTEMS ${ }^{1}$
}

\author{
Ching-Liang Huang and Victor O.K. Li \\ Department of Electrical Engineering \\ University of Southern California \\ Los Angeles, CA 90089-0272
}

\section{ABSTRACT}

Resilient commit protocols for multisite simple network partitioning are studied in this paper. The necessity of termination protocols to make commit protocols resilient in multisite simple network partitioning is presented. A termination protocol that makes the threephase commit protocol resilient is designed. This protocol is valid even for transient network partitioning. The method can be generalized to design termination protocols for other commit protocols in multisite simple network partitioning.

\section{Introduction}

A distributed database system enjoys the potential advantage of providing higher availability and reliability. However, this advantage cannot be achieved unless database consistency is guaranteed in the event of failures. Several commit protocols have been proposed $[1,2,4,5,6,7]$ to achieve transaction atomicity in the event of site failures for any number of participating sites, and network partitioning for two participating sites. But none of them are resilient in multisite network partitioning, in which the number of participating sites is more than two.

A termination protocol is a protocol that is invoked to consistently terminate transactions when failures occur and render the continued execution of a commit protocol impossible. In this paper, the necessity of termination protocols to make commit protocols resilient in multisite simple network partitioning is presented. A termination protocol that makes the three-phase commit protocol resilient is designed and the proof of its correctness is given. The protocol is valid even for transient network partitioning. This method may be generalized to design termination protocols for other commit protocols in multisite simple network partitioning.

\footnotetext{
${ }^{1}$ This research is supported in part by the Joint Services Electronics Program under Contract No. F49620-85C-0071, and in part by the National Science Foundation under Grant No. DCl8519101.
}

\section{Background}

A distributed database system supports a database physically distributed over multiple sites interconnected by a computer network. In such a system, there may be more than one site participating in a transaction. Since, by definition, a transaction is a logically atomic operation, to maintain database correctness, transaction atomicity must be enforced. That is, for any given transaction, either the transaction is processed to completion at all participating sites or it appears to have never been executed at all.

Preserving transaction atomicity in the single site case is a well understood problem $[1,3]$. When the site has finished processing the transaction but has not yet updated the database, it will decide to commit or abort the transaction (possibly by asking the user). If a commit decision is made, a commit log which contains the current state of the transaction (e.g. the update information) will be stored in stable storage, and then the site will start committing the transaction. If failures occur at any time before the commit log is stored, then immediately upon recovery the site will abort the transaction. If failures occur after the commit log is stored but before the updates are finished, all the updates will be applied again when the site recovers. Because update operations are idempotent (i.e. performing them several times is equivalent to performing them once), the above scheme ensures the atomicity of the transaction.

In the multiple site case, the problem of guaranteeing transaction atomicity is much more difficult because of arbitrary site failures and partitioning of the computer network. To ensure transaction atomicity in this case, each participating site performs either all or none of the updates locally. In addition, all the sites should make the same decision with respect to committing or aborting the transaction.

Protocols for preserving transaction atomicity are called commit protocols. Several commit protocols have been proposed $[1,2,4,5,6,7]$. The two phase commit protocol [1, 2] is the simplest one (Fig. 1). It is a centralized protocol with a single master and with the remaining participating sites acting as slaves. A participating site can be in one of the following four states 
: the initial state (q), the wait state (w), the commit state (c) and the abort state (a). The first phase of the protocol begins when the master receives the transaction ("request") and forwards it to the slaves ("Xact"). When a slave receives the transaction, it will partially execute the transaction and send its intent to commit ("yes") or unilaterally abort ("no") the transaction. The second phase begins when the master receives all the responses from the slaves. If all the participating sites agree to commit the transaction, then the master will send out commit commands ("commit") to the slaves, else it will send out abort commands ("abort"). When a slave receives the command, it will act accordingly.

The two phase commit protocol is simple. However, if the master fails and all the operating slaves are in the wait state, the slaves cannot make any decision to commit or abort the transaction (because the master can be in either the commit state or the abort state) and therefore must block the transaction until the failures recover. Even though blocking preserves database consistency, it is highly undesirable because the locks acquired by the blocked transaction cannot be relinquished, rendering those data inaccessible to other transactions. In [4], nonblocking commit protocols were studied, and a three-phase commit protocol and a termination protocol were presented which are nonblocking under site failures. Network partitioning was studied in [7], and some definitions and results from that paper are reviewed next.

A formal model is used to describe commit protocols. Transaction execution at each site is modelled as a finite state automaton (FSA), with the network serving as a common input/output tape to all sites. The states of the FSA for site $i$ are called the local states of site $i$. The global state of a distributed transaction consists of (1) a global state vector containing the local states of the participating sites, (2) the outstanding messages in the network. During a global state transition, there is exactly one local state transition, which involves a site reading a nonempty string of messages addressed to it, writing a string of messages, and moving to the next local state.

A commit protocol is resilient to a class of failures only if the protocol enforces transaction atomicity and is nonblocking for any failure within that class.

Network partitioning is divided into two classes:

1. simple partitioning in which the sites are partitioned into exactly two sets with no communication between the sets.

2. multiple partitioning in which the sites are partitioned into more than two sets.

Two models are considered for network partitioning:

1. pessimistic model where all messages are lost at the time partitioning occurs.
2. optimistic model where no messages are lost at the time partitioning occurs ; instead, undeliverable messages are returned to the sender.

Some results on the existence of resilient commit protocols for network partitioning have been developed.

Theorem: There exists no protocol resilient to a network partitioning when messages are lost.

Theorem: There exists no protocol resilient to a multiple network partitioning.

For two-site simple partitioning with return of messages (optimistic model), some results have been derived.

Definition: Concurrency Set $C(s)$ Let $s$ be an arbitrary local state of a commit protocol $P$. The concurrency set of $S$ is the set of all local states that are potentially concurrent with it in the execution of $P$.

Definition: Sender Set $S(s)$ Let $s$ be an arbitrary local state of a commit protocol $P$, and let $M$ be the set of messages that can be received by $s$ in the execution of $P$. $S(s)=\{t \mid t$ sends $m, m \varepsilon$ M \}

Two rules are defined for designing resilient commit protocols for two-site simple partitioning with return of messages.

Rule(a): For a state $s_{j}$ : if its concurrency set, $C\left(s_{j}\right)$, contains a commit state, then assign a timeout transition from $s_{i}$ to a commit state; else assign a timeout transition from $s_{i}$ to an abort state.

Rule(b): For state $s_{j}$ : if $t_{i}$ is in $S\left(s_{j}\right)$ and $t_{i}$ has a timeout transition to a commit (abort) state, then assign an undeliverable message transition from $s_{j}$ to a commit (abort) state, upon the receipt of an undeliverable message.

Using the above two rules, an extended two-phase commit protocol augmented with timeout transitions and undeliverable message transitions is derived (Fig. 2). It has been proved that these two rules are necessary and sufficient for making protocols resilient to two-site simple partitioning with return of undeliverable messages [7].

\section{Motivation}

The extended two-phase commit protocol shown above works correctly in optimistic two-site simple partitioning; however, we note that it does not work in the multisite case by the following observation:

Consider a transaction with three participating sites where site $_{1}$ is the master. Suppose a partitioning occurs 
with a global state vector of $\left\langle\mathrm{p}_{1}, \mathrm{w}_{2}, \mathrm{w}_{3}\right\rangle$ and outstanding messages $<-$, commit $_{2}$, commit $_{3}>$, i.e. the master is in the prepare state, the slaves are in the wait state and the master has sent out commit messages. If the partitioning causes site $_{3}$ to be separated from site ${ }_{1}$ and site $_{2}$, and commit $_{3}$ undeliverable, then site 2 will receive commit 2 and commit while site $_{3}$ will make a timeout transition and abort.

The extended two-phase commit protocol does not work in the multisite case due to the following facts:

1. The wait state (w) of a slave contains both a commit and an abort in its concurrency set.

2. There exists a local state (the wait state of a slave) that is noncommittable and contains a commit in its concurrency set.

( A local state is called committable if occupancy of that state by any site implies that all sites have voted yes on committing the transaction. Otherwise, it is called noncommittable [4].) lemmas.

From the above observations, we get the following

Lemma 1: A commit protocol $P$ can be made resilient to optimistic multisite simple network partitioning only if there does not exist a local state $S$ in $P$, such that the concurrency set of $s$ contains both a commit and an abort state.

Proof: Assume there exists a local state $s$ of site $_{i}$ that contains both a commit and an abort in its concurrency set $C(s)$. If site $_{i}$ is separated from the other participating sites by the network partitioning when it is in state $s$ and it has not obtained any information about whether the other sites commit or abort the transaction, then as long as the network is partitioned, site ${ }_{i}$ cannot tell whether the other sites commit or abort the transaction. Therefore site $_{i}$ must be blocked; else no matter whether site $_{\mathbf{i}}$ commits or aborts the transaction before the network recovers, the database would probably become inconsistent.

Q.E.D.

Lemma 2: A commit protocol $P$ can be made resilient to optimistic multisite simple network partitioning only if there does not exist a local state $s$, such that $s$ is noncommittable and the concurrency set of $s$ contains a commit state.

Proof: Assume there exists a local state of site ${ }_{i}$ that is noncommittable and contains a commit in its concurrency set. If site $_{i}$ is separated from the other participating sites by the network partitioning when it is in state $s$ and all messages addressed to site $e_{i}$ become undeliverable, then site ${ }_{i}$ should not commit because it cannot ensure that all the other participating sites agree to commit. However, site $e_{i}$ should not abort either because some participating site may have committed already. Therefore, site i must be blocked.

Q.E.D.

The two lemmas above is similar to the Fundamental Nonblocking Theorem in [4] which considers site failures instead of network partitioning.

The three-phase commit protocol [4] (Fig. 3) satisfies both Lemma1 and Lemma2; however, we found that augmenting this protocol by timeout transitions and undeliverable message transitions using Rule(a) and Rule(b) shown in Sec. 2 does not make it resilient to optimistic multisite simple network partitioning by the following observation:

Consider a transaction of three participating sites where site $_{2}$ and site $_{3}$ are slaves, and abort $\varepsilon \quad C\left(w_{3}\right)$, commit $\varepsilon C\left(p_{2}\right), p_{2} \varepsilon C\left(w_{3}\right)$. By Rule(a), the timeout transition from $w_{3}$ should go to the abort state and the timeout transition from $p_{2}$ should go to the commit state If site $_{3}$ is in state $w_{3}$ waiting for prepare $_{3}$ and site $_{2}$ is in state $\mathrm{p}_{2}$ waiting for commit 2 when partitioning occurs which renders prepare ${ }_{3}$ undeliverable, then site $_{3}$ will timeout and abort while site $_{2}$ will timeout and commit. Therefore, the database becomes inconsistent.

\section{Necessity of termination protocol}

The three-phase commit protocol is the simplest commit protocol that satisfies both Lemma1 and Lemma2. However, it cannot be made resilient to optimistic multisite simple network partitioning by simply augmenting it with timeout and undeliverable message transitions. If we add some more phases to our commit protocol, will timeout and undeliverable message transitions be sufficient to make the protocol resilient ? The following lemma yields a negative answer.

Lemma 3: If a commit protocol is not resilient to optimistic multisite simple network partitioning, then timeout transitions and undeliverable message transitions are not sufficient for making it resilient.

Proof: It has been proved that protocols in which each state transition reads at most one message are equivalent in power to more general protocols that read an arbitrary number of messages per state transition [6]. Therefore, we only consider protocols of the first type in this proof.

Assume network partitioning will cause all outstanding messages transmitted in the network at the time of partitioning between pairs of sites located in different partitions to be returned to the senders, i.e. Let $m_{i j}$ be the message sent by site $e_{i}$ to site $e_{j}$. If site $e_{i}$ and site $e_{j}$ are located in different partitions and $m_{i j}$ are outstanding when partitioning occurs, then $m_{i j}$ will be returned to site $e_{i}$.

Suppose the network is divided into two partitions: 
$G_{1}$ and $G_{2}$. We can always name the participating sites of a transaction such that all the sites in $G_{1}$ precede all the sites in $G_{2}$. i.e. site $i$ is in $G_{1}$ for $1 \leq i \leq k$, site $j$ is in $G_{2}$ for $k+1 \leq j \leq n$ where $n$ is the number of participating sites, $n$ $>2$, and $1 \leq k<n$.

Assume there exists a commit protocol $P$ that is not resilient to optimistic multisite simple network partitioning and can be made resilient by augmenting it with only timeout and undeliverable message transitions. Let $H^{0} H^{1}$ ... $H^{c}$ be the global state sequence of a failure-free execution of $P$ that commits a transaction.

Let $H^{i}=\left(S^{i}, M^{i}\right)$ where the global state vector $s^{i}=\left(s_{1}{ }^{i}, \ldots, s_{n}{ }^{j}\right)$, and the outstanding messages:

$$
\begin{aligned}
& M^{i}=m_{1,1^{i}} \quad \ldots m_{1, k}^{i} \quad m_{1, k+1}{ }^{i} \quad \ldots m_{1, n}{ }^{i} \\
& \begin{array}{llllll}
m_{k, 1} i^{i} \quad \ldots & m_{k, k}{ }^{i} \quad m_{k, k+1} i^{i} \quad \ldots & m_{k, n}{ }^{i}
\end{array} \\
& \begin{array}{llllll}
m_{k+1,1} & \ldots & m_{k+1, k}{ }^{i} m_{k+1, k+1}{ }^{i} & \ldots & m_{k+1, n}
\end{array} \\
& m_{n, 1} i^{i} \quad \ldots m_{n, k}{ }^{i} \quad m_{n, k+1}{ }^{i} \quad \ldots m_{n, n}{ }^{i}
\end{aligned}
$$

$m_{p, q} i$ is the set of outstanding messages in the network sent by site $_{p}$ to site $q$ when the global state is $H^{i}$.

Let $U D\left(m_{p, q_{i}}\right)$ denote the set of undeliverable messages of $m_{p, q}^{p, q_{i}}, \underline{H}^{i}=\left(S^{i}, \underline{M}^{i}\right)$ be the global state resulting from a partitioning that occurs during global state $H^{i}$.

$$
\begin{aligned}
& \underline{M}^{i}=x_{1,1}^{i} \quad \ldots \quad m_{1, k}^{i} \quad \phi \quad \ldots \quad l \\
& \begin{array}{llllll}
m_{k, 1} i^{i} & \ldots & x_{k, k}{ }^{i} \quad \phi \quad \ldots & \phi
\end{array} \\
& \phi \quad \ldots \quad \phi \quad x_{k+1, k+1} i^{i} \quad \ldots \quad m_{k+1, n} \\
& \begin{array}{lllllll} 
& \ldots & \phi & m_{n, k+1}{ }^{i} & \ldots & x_{n, n}{ }^{i}
\end{array}
\end{aligned}
$$

where $x_{f, f}^{i}=m_{f, f^{i}} \quad \cup \quad U D\left(m_{f, k+1}{ }^{i}\right) \cup \ldots \cup \cup D\left(m_{f, n}{ }^{i}\right)$

for $f=1$ to $k$

$$
m_{f, f}{ }^{i} \cup \quad \cup D\left(m_{f, 1^{\prime}}\right) \quad \cup \ldots \cup \cup D\left(m_{f, k}{ }^{i}\right)
$$

for $f=k+1$ to $n$

Let $\underline{M}_{j}^{i}=\operatorname{COLUMN}_{j}\left(\underline{M}^{i}\right)$.

Suppose $F_{j}$ is the transition function that moves site to a final state during partitioning, i.e. $F_{j}\left(s_{j}{ }^{i}, M_{j}{ }^{i}\right)$ is the resulting final state of site $e_{j}$ when partitioning occurs during $\mathrm{H}^{\mathrm{i}}$.
Let $r$ be the smallest $i$ such that a simple partitioning which occurs while the transaction is in $H^{i}$ results in the transaction being committed, i.e. $F_{j}\left(s_{j}{ }^{r}, M_{j}^{r}\right)=$ commit for $j$ $=1$ to $n$. By the choice of $r$, we have $F_{j}\left(s_{j}^{r-1}, M_{j}^{r-1}\right)=$ abort for $j=1$ to $n$ for the same partitioning.

Let the global transition from $\mathrm{H}^{\mathrm{r}-1}$ to $\mathrm{H}^{\mathrm{r}}$ be made by site $e_{p}$ reading a message $u_{q p}$ sent by site $q_{q}$ and sending some (null or nonnull) messages $\left\{u_{p 1} \ldots u_{p n}\right\}$. Therefore,

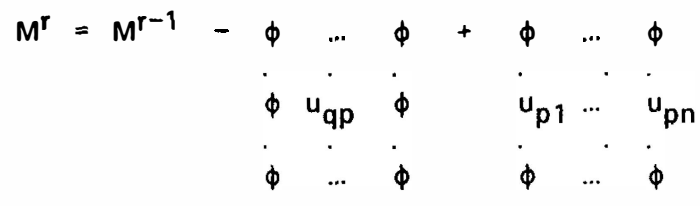

We consider the following four cases :

(1) $1<q<k, 1<p<k$ then $\underline{M}_{j}^{r}=\underline{M}_{i}^{r-1}$ for $k+1 \leq j \leq n$

(2) $1 \leq q \leq k, k+1 \leq p \leq n$ then $\underline{M}_{j}^{r}=\underline{M}_{j}^{r-1}$ for $1 \leq j \leq k, \quad j \neq q$

(3) $k+1 \leq q \leq n, 1 \leq p \leq k$

$$
\text { then } \underline{M}_{j}^{r}=M_{j}^{r-1} \text { for } k+1 \leq j \leq n, \quad j \neq q
$$

(4) $k+1 \leq q \leq n, k+1 \leq p \leq n$

$$
\text { then } \underline{M}_{j}^{r}=\underline{M}_{j}^{r-1} \text { for } 1 \leq j \leq k
$$

Since only site $p_{p}$ makes a transition during the global transition from $H_{r-1}$ to $H_{r}, s_{j}{ }^{r}=s_{j}^{r-1}$ for $j \neq p$. So in all cases, there exists a site ${ }_{j}$ such that $s_{j}{ }^{r}=s_{j}{ }^{r-1}$ and $M_{j}{ }^{r}=$ $\underline{M}_{j}^{r-1}$. Hence, $F_{j}\left(s_{j}^{r-1}, M_{j}^{r-1}\right)=F_{j}\left(s_{j}^{r}, \underline{M}_{j}^{r}\right)$ which is a contradiction.

Therefore, there does not exist such a protocol P.

Q.E.D.

\section{A termination protocol for three-phase commit in multisite simple network partitioning}

Since timeout transitions and undeliverable message transitions are not sufficient to make commit protocols resilient to multisite simple partitioning, we need to invoke another protocol to consistently terminate the transactions when partitioning occurs. This protocol is called a termination protocol. In this section, we present a termination protocol that makes the three-phase commit protocol resilient to optimistic multisite simple network partitioning 


\subsection{Assumptions}

We make several assumptions for the following discussion:

1. all undeliverable messages due to network partitioning are returned to the sender.

2. there is no subsequent network partitioning before all the transactions affected by the previous partitioning have terminated, i.e. there is no multiple network partitioning.

3. network partitioning and site failures never occur concurrently.

4. masters never fail.

5. once the network is partitioned, it will remain in the partitioned state until all the transactions affected by the partitioning have terminated, i.e. there is no transient network partitioning.

Assumption 5 simplifies the problem but will be relaxed later.

\subsection{Approach to the problem}

Denote the two partitions of the network $G_{1}, G_{2}$ and the boundary between them B (Fig. 4). A site can tell whether partitioning has occurred or not by having a timeout or receiving an undeliverable message. Note, however, that a site does not know which partition it is in and where the boundary is. For a particular transaction, let $G_{1}$ be the partition in which the master is located. The basic ideas for solving the problem are as follows :

1. Slaves in $G_{2}$ will commit iff there is at least one prepare message flowing through boundary $B$ before partitioning occurs. If there exists such a prepare message, then the master can commit all the slaves in $G_{1}$ and the slave ( or slaves, since there may be more than one) in $G_{2}$ that receives the prepare message will be responsible for committing all the slaves in $\mathbf{G}_{2}$.

2. If partitioning occurs when the master is in $w_{1}$ state waiting for "yes" and if the master times out eventually, then the master can safely abort all the slaves in $G_{1}$ since no prepare messages have been generated and none of the slaves in $\mathrm{G}_{2}$ will commit.

3. If partitioning occurs when the master is in $p_{1}$ state waiting for "ack" and if the master times out eventually and there is no undeliverable prepare message returned, then the master can safely commit all the slaves in $G_{1}$ since we are sure that all the slaves will receive the prepare message and all the slaves in $G_{2}$ will commit.

4. If partitioning occurs when the master is in $p_{1}$ state and there is at least one undeliverable prepare message returned to the master, then the master can tell whether there is at least one prepare message flowing through boundary $B$ before partitioning occurs by the following rules :

a. When a slave times out in p(prepare) state, it will send a probe message: probe(trans_id, slave_id) to the master.

b. If the probe messages that the master received are sent by exactly those slaves that do not have an undeliverable prepare message returned to the master then there is no prepare message flowing through boundary $B$ and the master can safely abort all the slaves in $\mathbf{G}_{1}$ else there is at least one prepare message flowing through boundary $B$ and the master can safely commit all the slaves in $\mathbf{G}_{1}$.

5. If partitioning occurs when the master is in $p_{1}$ state and there is at least one undeliverable prepare message returned to the master, then all the slaves in $G_{1}$ will time out in $p$ state eventually.

6. A slave which has received a prepare message can tell that it is in $G_{2}$ by (1) receiving a returned undeliverable "ack" message, or (2) receiving a returned undeliverable probe message. Such a slave will commit all the slaves in $\mathrm{G}_{2}$.

\subsection{The termination protocol}

In this subsection, we describe the termination protocol by specifying the actions to be taken when timeout occurs or an undeliverable message is received in each state.

\section{Notations}

abort $_{1-n}: \quad\left\{\right.$ abort $_{1}, \ldots$ abort $\left._{n}\right\}$

commit $_{1-n}: \quad\left\{\right.$ commit $_{1}, \ldots$, commit $\left._{n}\right\}$

UD( msg ): the corresponding undeliverable message of $\mathrm{msg}$.

PB: $\quad$ the set of slaves from which the master has received probe messages.

UD: the set of slaves to which the prepare messages sent by the master are undeliverable.

T

the longest end-to-end network propagation delay.

$\mathbf{N}$ transaction $=\{1,2, \ldots, n\}$. 


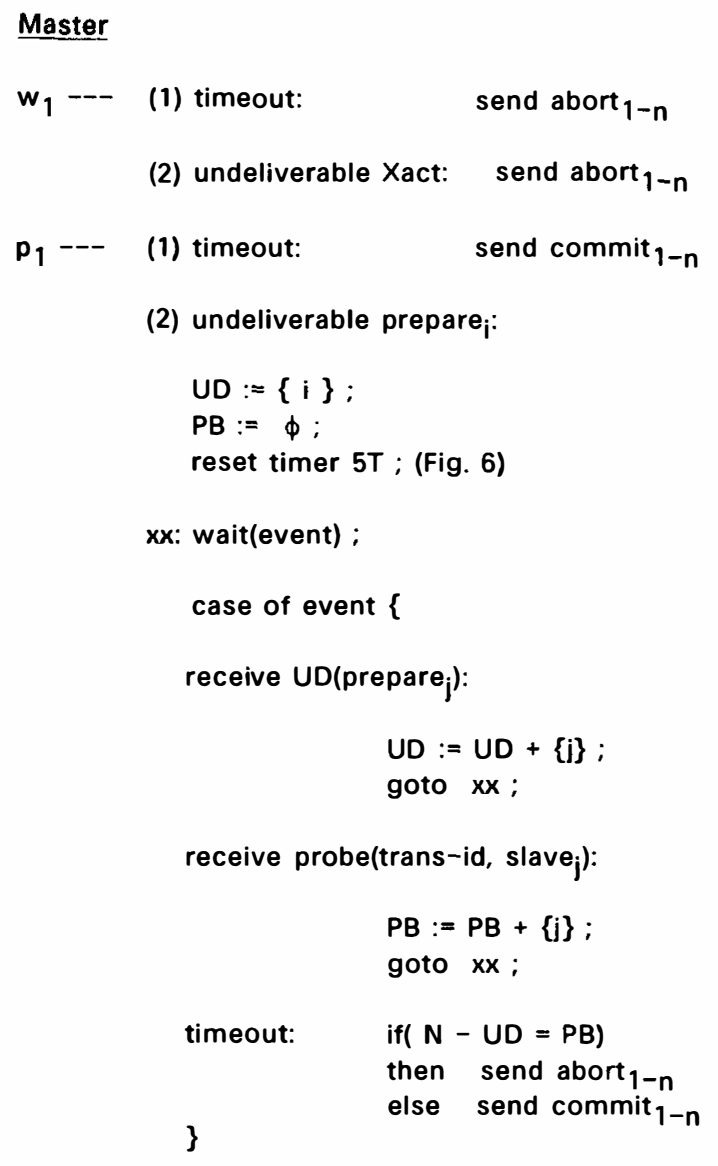

Slaves $(i=2$ to $n)$

$w_{i}---(1)$ timeout: reset timer $6 \mathrm{~T} ;($ Fig. 7$)$ wait(event) : case of event \{

receive a commit: commit ; receive an abort: abort ; timeout: abort \}

(2) undeliverable yes ${ }_{j}$ : send abort $1-n$;

\footnotetext{
$p_{i}--(1)$ timeout: send probe(trans-id, slave $\left.e_{j}\right)$; wait(event) ; case of event \{

(2) undeliverable Ack: send commit $1-n$;

Unfortunately, there is still a fly in the ointment. Consider the following scenario : Let site $_{j}$ and site $_{j}$ be two of the sites in $G_{2}$. Site $e_{i}$ receives a prepare message, sends out an "ack" message and receives a returned undeliverable ack message. Site $e_{j}$ receives no prepare message and waits

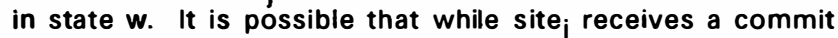
sent by site $_{j}$, site $e_{j}$ is still in state $w$ and has not timed out yet; therefore, site $j_{j}$ will not respond to this commit message. This may cause a serious problem because the commit message could be the only commit message site will ever receive. If it misses this message, after it times out in state $w$ and waits for $6 T$, it will abort the transaction. To deal with this problem, we can add one transition from state $w$ to state $c$ when receiving a commit message to the slaves' three-phase commit protocol (Fig. 8).

\subsection{Proof of Correctness}

In this subsection, we give a formal proof of correctness of the termination protocol.

FACT1: A slave in $\mathrm{G}_{2}$ will commit only if

(1) it receives a commit from the master,

(2) it times out in $w_{i}$, and then receives a commit,

(3) it times out in $p_{i}$, and then receives an UD(probe),

(4) it times out in $p_{i}$, and then receives a commit,

(5) it receives an UD $\left(a c k_{i}\right)$ in $p_{i}$, or

(6) it receives a commit from some slave in $G_{2}$ while it is in state $w$ or state $p$.

FACT2: A site in $G_{1}$ will commit only if

(1) the master makes a transition from $p_{1}$ to $c_{1}$ and sends out all the commits,

(2) the master times out in $p_{1}$ and sends out all the commits, or

(3) the master receives an UD(prepare ${ }_{j}$ ), finds that $N-U D \neq P B$ and sends out all the commits.

Lemma 4: $N$ - UD $\neq$ PB occurs only if at least one slave in $G_{2}$ receives a prepare message.

Proof: The master will check whether $\mathbf{N}-$ UD $=$ PB only if it sends out all the prepare messages and receives an UD(prepare) message. Therefore, all the slaves in $G_{1}$ will receive prepare messages.

If none of the slaves in $G_{2}$ receives a prepare message, then only those slaves in $G_{1}$ receive prepare messages. The slaves in $G_{1}$ will time out and send probe messages which will be received by the master. Therefore, $\mathbf{N}-\mathrm{UD}=\mathrm{PB}=$ set of all slaves in $\mathbf{G}_{1}$.

Q.E.D.

Lemma 5: A slave in $G_{2}$ commits iff all slaves in $G_{2}$ commit. 
Proof: "if" : trivial.

"only if" : Let the slave in $G_{2}$ that commits be site $e_{j}$. By FACT1, we know that there are only six possible cases.

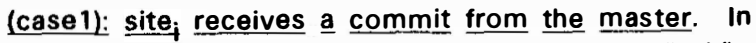
this case, the master must have received all the "ack"s, made a transition from $p_{1}$ to $c_{1}$ and sent out all the commits. Therefore, for any site $\mathrm{i}_{\mathrm{j}}$ in $\mathrm{G}_{2}$ either it will time out in $p_{j}$ or commit in $c_{j}$. If it times out in $p_{j}$, then it will eventually receive the returned UD(probe) message and commit all the sites in $G_{2}$.

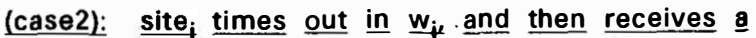
commit. Since site $e_{i}$ is still in $w_{j}$, the commit it receives must come from some slave $e_{j}$ in $G_{2}$. When a slave in $G_{2}$ sends out commits (after receiving an UD(probe) or UD(ack) ), it will send to all the slaves in $G_{2}$. Therefore, all the slaves in $G_{2}$ will commit.

(case3): site ${ }_{i}$ times out in $\mathbf{p}_{i}$ and then receives an UD(probe). When site ${ }_{i}$ receives a UD(probe), it will send commits to all slaves in $G_{2}$; therefore, all slaves in $G_{2}$ will commit.

(case4): site $e_{i}$ times out in $p_{i}$ and then receives a commit. Since site $e_{i}$ receives a commit after it times out in $p_{i}$, this commit message must come from some slave in $G_{2}$. By the same argument as (case2), all slaves in $G_{2}$ will commit.

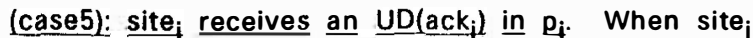
receives an $U D\left(a c k_{i}\right)$, it will send commits to all slaves in $G_{2}$; therefore, all slaves in $G_{2}$ will commit.

(case6): site $_{\text {i }}$ receives a commit from some slave in $\underline{G}_{2}$ while it is in state $\underline{w}$ or state $p$. By the same argument as (case2), all slaves in $G_{2}$ will commit.

Q.E.D commit.

Lemma 6: A site in $G_{1}$ commits iff all sites in $G_{1}$

Proof: "if" : trivial.

"only if" : By FACT2, if a site in $G_{1}$ commits, then all sites in $G_{1}$ will commit.

\section{Q.E.D.}

Lemma 7: A slave in $G_{2}$ receives a prepare message iff all slaves in $G_{2}$ commit.

Proof: "if" : trivial.

"only if": Let the slave in $G_{2}$ that receives a prepare message be site ${ }_{\mathbf{j}}$. There are three possible cases.

(case1) site receives a commit too. By Lemma5, all the slaves in $G_{2}$ will commit. (case2) site $_{i}$ times out in $\mathrm{P}_{i}$. Site $\mathrm{e}_{\mathrm{i}}$ will probe the master and eventually it will receive the returned UD(probe) or a commit sent by some slave in $G_{2}$ and commit. By Lemma5, all slaves in $G_{2}$ will commit.

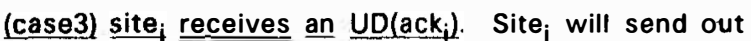
commits to all slaves in $G_{2}$; therefore, all slaves in $G_{2}$ will commit.

Q.E.D.

Lemma 8: A slave in $G_{2}$ receives a prepare message iff all sites in $G_{1}$ commit.

Proof: "only if" : Let the slave in $G_{2}$ that receives a prepare be site . Since site $_{i}$ has received a prepare message, the master must be in $p_{1}$ or $c_{1}$. If the master is in $c_{1}$, then all the sites in $G_{1}$ will commit by Lemma6.

If the master is in $p_{1}$, there are two cases to be considered.

(case 1): The master times out in $p_{7}$. The master will commit and send commits to all the slaves; therefore, all sites in $G_{1}$ will commit.

(case2): The master receives an UD(prepare) message. Since site ${ }_{i}$, located in $G_{2}$, has received a prepare message and the master can only receive the probes sent by slaves in $G_{1}$, the master will find that the set of slaves that probe it does not equal to the set of slaves that have received prepare messages. Therefore, the master will send commits to all slaves in $G_{1}$ and all of them will commit.

"if" : By FACT2, there are three cases to be considered.

(case 1): The master makes a transition from $p_{1}$ to $c_{1}$ and sends out all the commits. Since the master will make a transition from $p_{1}$ to $c_{1}$ only if it has received all the "ack" messages, all the slaves must have received the prepare message.

(case2): The master times out in $p_{1}$ and sends out all the commits. Since the master will time out in $p_{1}$ only if all the prepare pessages are deliverable, all the slaves must have received the prepare message.

(case3): The master receives an UD(prepare) message, finds that $\underline{N}=\underline{U D} \equiv \underline{P B}$ and sends out all the commits. By Lemma4, there exists a slave in $G_{2}$ which has received a prepare message.

Q.E.D.

Theorem 9: The termination protocol makes the three-phase commit protocol resilient to optimistic multisite simple network partitioning.

Proof: By Lemma5 to Lemma8, we know that 
(1) a slave in $G_{2}$ commits implies all the slaves in $G_{2}$ commit which implies a slave in $G_{2}$ receives a prepare message which means that all sites in $G_{1}$ commit.

(2) a site in $G_{1}$ commits implies all sites in $G_{1}$ commit which implies a slave in $G_{2}$ receives a prepare which means that all slaves in $G_{2}$ commit.

Therefore, the three-phase commit protocol becomes resilient to optimistic multisite simple network partitioning

Q.E.D.

In fact, the basic ideas used in designing the above termination protocol can be applied to design termination protocol for any master-slave type commit protocol that satisfies Lemma1 and Lemma2.

Theorem 10: A commit protocol can be made resilient to multisite simple network partitioning if the following conditions are satisfied:

1. there exists no local state that has both a commit and an abort in its concurrency set.

2. there exists no local state that is noncommittable and has a commit in its concurrency set.

3. undeliverable messages are returned to the senders.

4. there is no concurrent network partitioning and site failures.

5. masters never fail.

Proof: For any commit protocol that satisfies conditions (1) and (2), the only adjacent states of a commit state must be committable states and these committable states cannot be adjacent to an abort state. To commit a transaction in such a protocol, there exists a message sent by the master for a slave to make a transition from a noncommittable state to a committable state. Let this message be " $\mathrm{m}$ ". We can use the ideas listed in Sec. 5.2 to design a termination protocol for this commit protocol in an environment that satisfies conditions (3), (4) and (5) by substituting " $\mathrm{m}$ " for "prepare".

Q.E.D.

\section{Transient Network Partitioning}

A network partitioning is transient if the network recovers before all the transactions affected by the partitioning have terminated. In the previous discussion, we assume that transient network partitioning never occurs in order to simplify the discussion. In this section, we will relax this assumption.

If we take transient network partitioning into consideration, we can enumerate all the possible cases of network partitioning as follows:
(1) no prepare messages pass boundary $B$.

(2) some prepare messages pass B, some prepare messages do not pass $B$. This case can be divided into the following two cases :

(2.1) some ack messages do not pass B.

(2.2) all the ack messages sent by those sites in $G_{2}$ which receive prepare messages pass $B$. This case can be further divided into the following two cases for transient network partitioning:

(2.2.1) network recovers at a point in time such that some probe messages do not pass $B$.

(2.2.2) network recovers at a point in time such that all the probe messages sent pass $B$.

(3) all the prepare messages pass $B$.

This case can be divided into the following two cases:

(3.1) some ack messages do not pass B.

(3.2) all the ack messages pass $B$. This case can be further divided into the following two cases:

(3.2.1) all the commit messages pass $B$.

(3.2.2) some commit messages do not pass B. This case can be further divided into the following two cases for transient network partitioning:

(3.2.2.1) some probe messages sent by those sites in $G_{2}$ that receive no commit messages do not pass $B$.

(3.2.2.2) all the probe messages sent by those sites in $G_{2}$ that receive no commit messages pass $B$.

Our original termination protocol works well in all cases except case (3.2.2.2). In this particular case, those sites in $G_{2}$ that receive no commit messages sent by the master will wait forever. To deal with this problem, we calculate the longest possible time for a slave to receive an UD(probe) message, a commit or an abort after it times out in state $p$ and get the following result:

Case(2.1): T.

Case(2.2.1): 4T.

Case(2.2.2): $5 \mathrm{~T}$ (Fig. 9).

Case(3.1): T.

Case(3.2.2.1): 4T.

Case(3.2.2.2): $\infty$.

Since only case(3.2.2.2) takes more than $5 \mathrm{~T}$, a slave can tell that case(3.2.2.2) has happened and commits itself if it has waited for 5T after it times out in state $p$ and receives neither an UD(probe) message nor a commit nor an abort. Therefore, to deal with transient network partitioning, we only have to modify the action to be taken when a slave times out in state $p$ as follows:

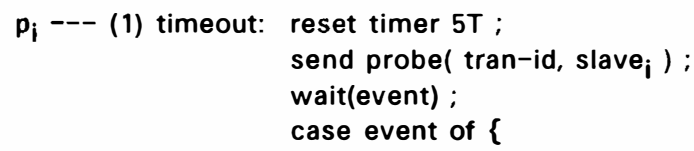




receive UD(probe): send commit $1-n$;
receive a commit: commit ;
receive an abort: abort :
timeout: $\quad$ commit
\}

\section{Conclusion}

In this paper, we assume that network partitioning and site failures never occur concurrently. This assumption is necessary due to the following two observations: (1) if the only slave in $G_{2}$ that receives a prepare message fails before it sends out commit messages, then all slaves in $G_{2}$ will abort while all participating sites in $G_{1}$ will commit. (2) if none of the slaves in $G_{2}$ receives a prepare message and one of the slaves in $G_{1}$ fails after receiving a prepare message but before sending a probe message, then all slaves in $G_{2}$ will abort while all participating sites in $G_{1}$ will commit. In fact, there is no commit protocol resilient to concurrent network partitioning and site failures because site failures in a network partitioning may have the same effect as message loss and it has been proved that there exists no commit protocol resilient to network partitioning when messages are lost [7]. Furthermore, we assume that masters never fail. The reason we make this assumption is that the termination protocol to be taken for network partitioning is different from the termination protocol to be taken for master site failure which has been proposed by Dale Skeen [4] and there is no simple way to distinguish a site failure from a network partitioning which separates that site from the rest of the system. These two assumptions amount to assuming that site failures never occur and a site will abort a transaction only because of

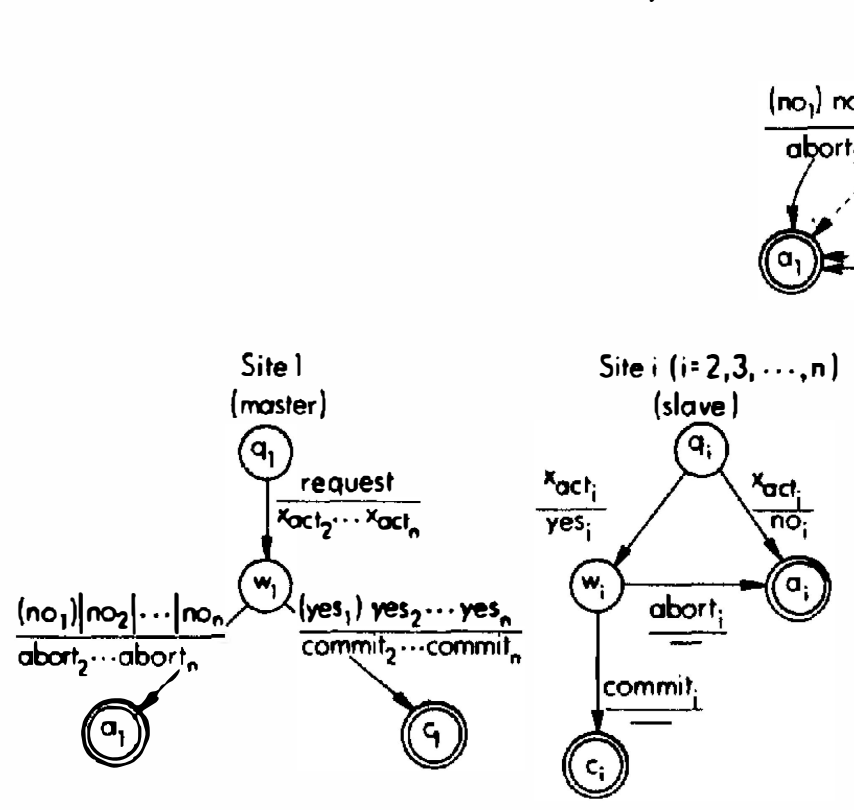

network failures, transaction deadlocks with other transactions or the user aborting the transaction. With technological advances of hardware fault tolerance, computers will be very reliable compared to links in the network. Therefore, we feel that assuming site failures never occur is acceptable.

\section{References}

1. J. Gray. Notes on database operating systems. in Operating System: An Advanced Cource. New York: Springer-Verlag, 1979.

2. B. Lampson, H. Sturgis. Crash Recovery in a Distributed Storage System. Computer Sci. Lab., Xerox Parc, Palo Alto, CA, 1976.

3. B. G. Lindsay et al. Notes on Distributed Databases. IBM Research, July, 1979.

4. D. Skeen. Nonblocking Commit Protocols. SIGMOD Int. Conf. on Management of Data, 1981, pp. 133-142.

5. D. Skeen. A Quorum-Based Commit Protocol. Proc. 6th Berkeley Workshop, February, 1982, op. 69-80.

6. D. Skeen. Crash Recovery in Distributed Database System. Ph.D. Th., Dept. of EECS, University of California, Berkeley, May 1982.

7. D. Skeen, M. Stonebraker. "A Formal Model of Crash Recovery in a Distributed System." IEEE Trans. On Sof tware Eng. SE-9, 3 (May 1983), 219-228.
Sile 1 (master)

(q)

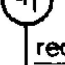
request
Site2

(slave)

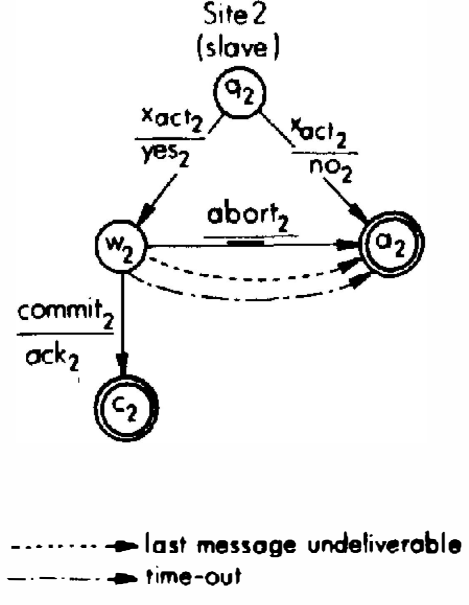

Figure 2: The extended two-phase commit protocol augumented with timeout transitions and undeliverable message transitions (two participating sites).

Figure 1: The two-phase commit protocol. 


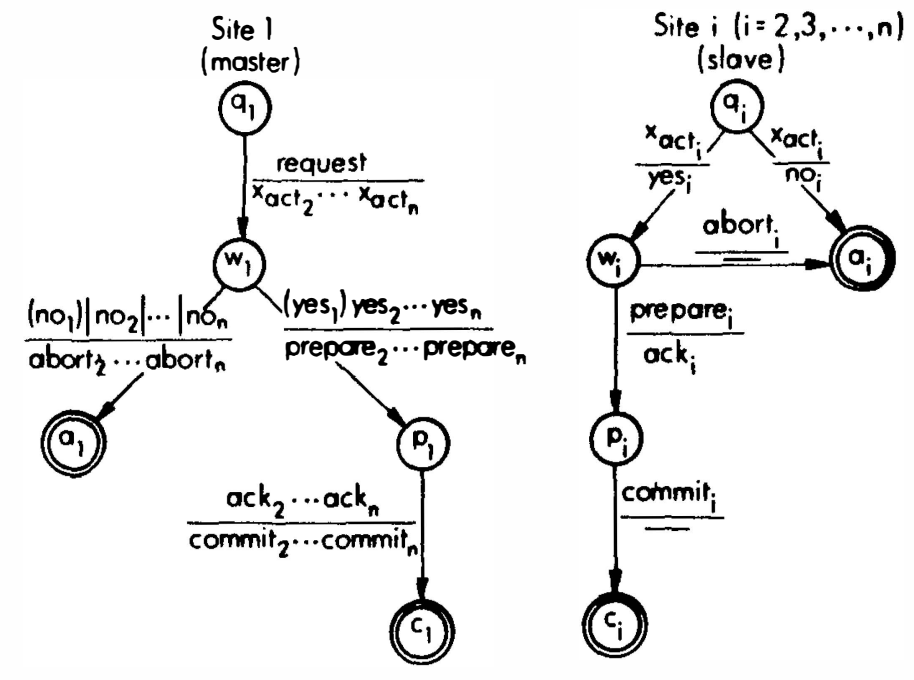

Figure 3: The three-phase commit protocol.

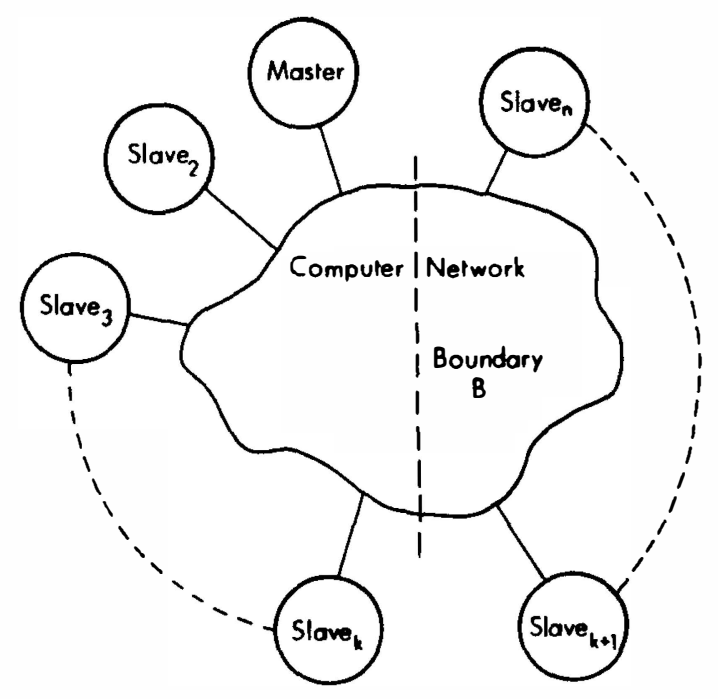

Figure 4: Simple network partitioning.

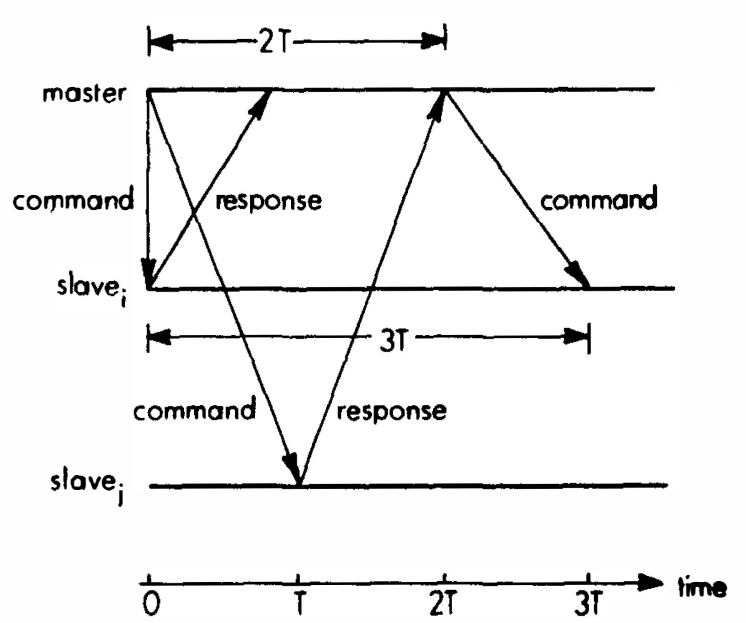

Length of timeout interval for the commit protocol at the master site $=2 \mathrm{~T}$.

Length of timeout interval for the commit protocol at slave sites $=3 \mathrm{~T}$.

( $T$ : the longest end-to-end network propagation delay )

Figure 5: Timeout analysis.

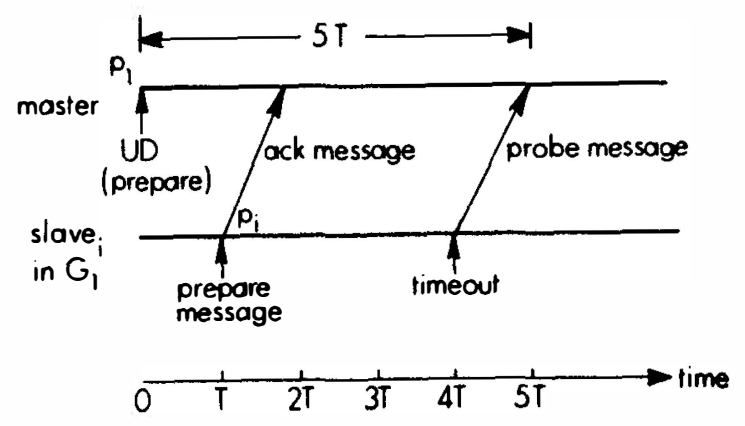

The longest possible time for a master to receive the probe message after receiving an undeliverable prepare message $=5 T$.

Figure 6: Timing analysis 


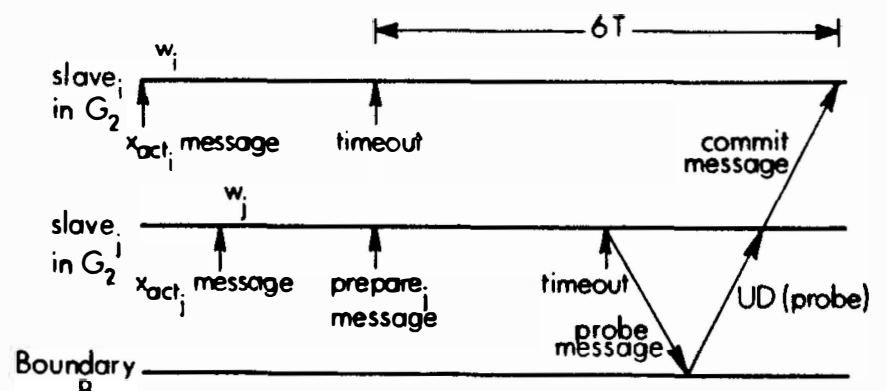

B
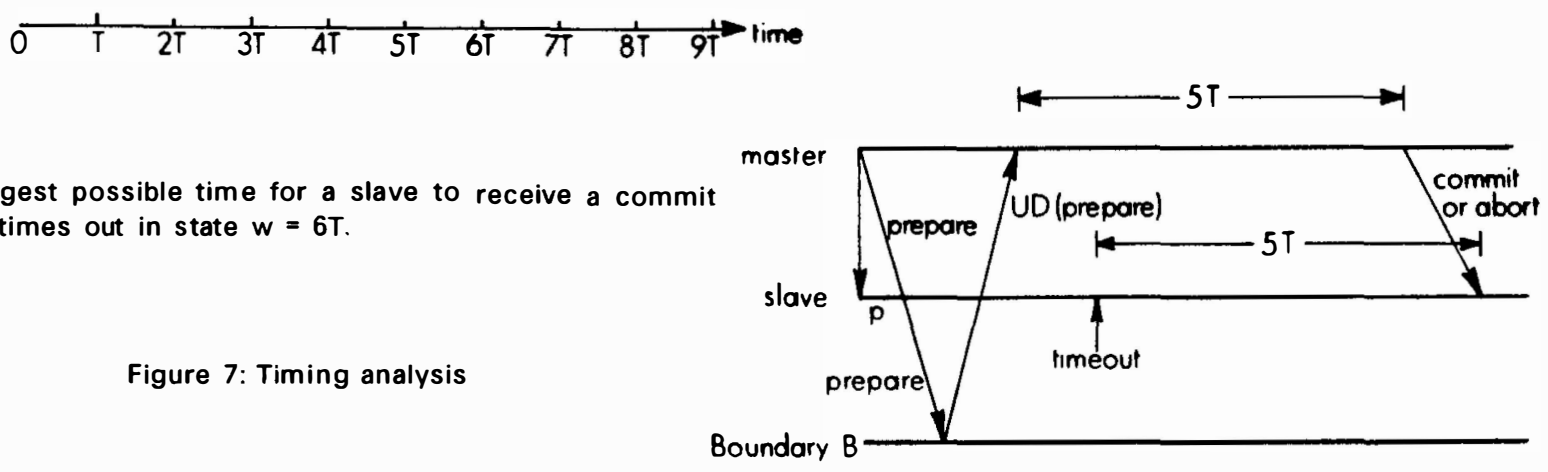

The longest possible time for a slave to receive a commit after it times out in state $w=6 T$.

Figure 7: Timing analysis

Boundary B

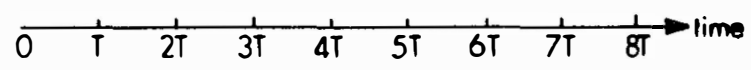

The longest possible time for a slave to receive an UD(probe) message, a commit or an abort after it times out in state $p=5 T$.
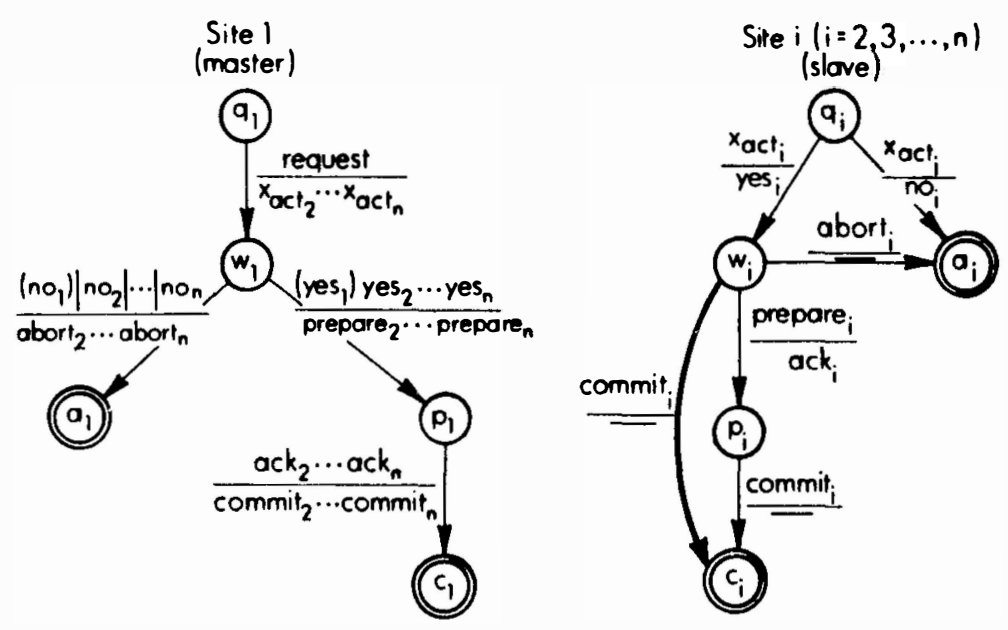

Figure 9: Timing analysis

Figure 8: The modified three-phase commit protocol 